\title{
Vault-1 - A Mission Architecture for Human Exploration of Near-Earth Objects
}

\author{
Jason Rabinovitch * \\ California Institute of Technology, Pasadena, California, 91125, USA \\ Ashish Goel ${ }^{\dagger}$ \\ Stanford University, Stanford, California, 94305, U.S.A. \\ Urszula Niedzielska ${ }^{\ddagger}$ \\ Warsaw University of Technology, Warsaw, 00661, Poland \\ Sriram Ganesan ${ }^{\S}$ \\ Indian Institute of Technology, Kanpur, Uttar Pradesh, 208016, India \\ Rachel E. Forman \\ Massachusetts Institute of Technology, Cambridge, Massachusetts, 02139, U.S.A.
}

In 2011, the Keck Institute for Space Studies hosted the Caltech Space Challenge, a week long workshop/competition directed towards the President's initiative of sending astronauts to an asteroid by 2025. Two teams composed of 16 students each, representing 12 different nations, competed to prepare a mission proposal by the end of the week. This report highlights some of the work done by Team Voyager. Not only is this work useful in showing that a manned mission to a Near-Earth Object is feasible by 2025, but it also demonstrates the utility of intense, relatively short student competitions.

This study is an outline of Vault-1, a proposed human mission to a Near-Earth Object. In addition to continuing human exploration, Vault-1 aims to make new discoveries about the origins of the Solar System, to develop technologies geared towards deep space exploration and a manned Mars mission, and to gain critical knowledge and experience to better protect Earth from future asteroid impacts. The primary target of this endeavor is 1999AO10, an asteroid that is larger than $30 \mathrm{~m}$ that also has an achievable mission duration of less than 200 days. 2000SG344 is a viable secondary target. Vault-1 will nominally carry 3 crew members to 1999AO10; after 14 days at the asteroid, the astronauts will return safely to the Earth with samples from the asteroid.

An official copy of the report Team Voyager created during the competition can be found at http://www.kiss.caltech.edu/study/space-challenge/Voyager_Final_Report.pdf

\section{Nomenclature}

$\begin{array}{ll}C P S & \text { Cryogenic Propulsion System } \\ D O R & \text { Differential One-Way Ranging } \\ D S H & \text { Deep Space Habitat } \\ D S V & \text { Deep Space Vehicle } \\ E V A & \text { Extra-Vehicular Activity }\end{array}$

*PhD Student, Graduate Aeronautical Laboratories, AIAA Student Member

$\dagger$ Doctoral Student, Department of Aeronautics and Astronautics

$\ddagger$ Graduate Student, Department of Aerospace Engineering

$\S$ Graduate Student, Department of Aerospace Engineering, AIAA Student Member

ฯ Graduate Student, Department of Aeronautics and Astronautics 
GCR Galactic Cosmic Ray

$H E O$ Highly Elliptical Orbit

$H L L V$ Heavy Lift Launch Vehicle

$I M F \quad$ Interplanetary Magnetic Field

$I_{S P} \quad$ Specific Impulse

LEO Low Earth Orbit

$M P C V$ Multi-Purpose Crew Vehicle

NEA Near-Earth Asteroid

$N E O \quad$ Near-Earth Object

NIR Near Infrared

PHO Potentially Hazardous Object

$S E V \quad$ Space Exploration Vehicle

$S E P \quad$ Solar Electric Propulsion

$S L S \quad$ Space Launch System

$S P E \quad$ Solar Particle Event

$S Q U I D$ Superconducting Quantum Interference Device

TIR Thermal Infrared

\section{Introduction}

NEAR-EARTh Objects (NEOs) are remnants from the formation of our Solar System and contain much useful information about the origins and creation of our Solar System. Exploration of these objects could explain how the Solar System was formed, and could give information on how the planets have evolved, and why they have the form they do today. Understanding NEOs, their structure, composition and trajectories could also help humans develop techniques to prevent a future collision of a Potentially Hazardous Object (PHO) with the Earth. NEOs are also potential sources of raw materials, including chemical elements such as iron, cobalt, platinum, osmium, volatiles and water. These objects could be used as a resource station for a deep space mission. A human mission to a NEO represents the first foray for furthering human exploration of the Solar System, and a stepping stone for the eventual goal of landing humans on Mars. A mission to a NEO will allow many deep space technologies to be tested and validated which will be required for a longer duration mission. NEOs can serve as a series of stepping stones spanning various distances between the Moon and Mars, allowing sustained technology improvements in small achievable steps, advancing our space technologies greatly.

This report presents a mission profile for Vault-1, a pioneering human mission to a Near-Earth Asteroid (NEA) with the objective of gaining valuable scientific knowledge about an asteroid and the Solar System, in addition to developing and testing technologies for advancing the frontiers of human space exploration. Designed with the desire to minimize cost, maximize benefits, and most importantly to keep the crew members safe, Vault-1 explores a variety of parameter spaces to make efficient use of state-of-the-art technologies, and technologies currently being developed, to meet the challenging requirements of the mission.

In the following sections, the various aspects of the mission are described in detail. In Section II, the choice of the asteroid and the design of the trajectory for the human sample return mission is discussed. In Section III, the propulsion solution for getting the astronauts to the asteroid and safely back to the Earth is described. In Section IV, the science experiments to be performed by the astronauts while at the asteroid are described. Next, in Section V, the issues associated with prolonged human presence in deep space are discussed and the conclusions reached from the mission design are presented in Section VI.

For the design of this mission, it is assumed that a precursor robotic mission will be launched 2-3 years before Vault-1 to image the chosen asteroid, characterize the radiation and dust environments at the asteroid, and gather other critical information for enabling the human mission. The robotic pre-cursor mission is not discussed in this mission proposal.

\section{Trajectory Planning}

The primary requirements for choosing the destination of this mission are to get the crew to the NEO, stay for a minimum of 10 days, and then return the crew and cargo safely to Earth. The entire duration 
of the mission is required to be less than 200 days, due to complexities introduced by having humans onboard. These objectives provided the major constraints for the target selection, trajectory design, propulsion systems, and spacecraft design.

\section{II.A. Target Selection}

There are several NEAs that are feasible candidates for the Vault-1 mission. As this is a manned mission, it is important to select an asteroid with a radius larger than approximately $30 \mathrm{~m}$, so that the spin rate is not so large that it restricts human interaction with the asteroid. ${ }^{1}$ Total $\Delta V$ required, launch opportunities, mission duration, and observation opportunities were also important selection criteria. In the preliminary selection process, objects 1999AO10, 2000SG344, 2004MN4, and 2001QJ142 all were potential targets, but 2004MN4 was discarded due to its extremely high $\Delta V$ requirement. The selection criteria for the three remaining asteroids are summarized in Table 1.

Table 1. Characteristics of candidate target asteroids. ${ }^{2}$

\begin{tabular}{cccccc} 
Asteroid & Size $(\mathrm{m})$ & $\Delta V(\mathrm{~km} / \mathrm{s})$ & Launch Year & Round-Trip Duration (days) & Observation Opportunity \\
\hline 1999AO10 & $45-100$ & 6.52 & 2025 & 180 & 2019 \\
2000SG344 & $30-66$ & 4.63 & 2029 & 180 & 2028 \\
2001QJ142 & $60-120$ & 6.73 & 2024 & 180 & 2012
\end{tabular}

1999AO10 was selected based on a number of positive characteristics. The asteroid 1999AO10 was chosen based on its size, lower $\Delta V$ requirement, and favorable launch opportunity. Another high-level goal during the design process of Vault-1 was to create a flexible design such that the infrastructure created for the Vault-1 Mission could be used for future manned missions. While 1999AO10 was chosen as the primary target, there are minimal changes necessary to go to 2000SG344, in case the launch window were to be missed, or if other unforeseen complications necessitated a target change. Even though 2001QJ142 has a possible observation opportunity, this is uncertain, and the higher $\Delta V$ requirement for 2001QJ142 made 1999AO10 the more attractive primary target and 2000SG344 the secondary target asteroid.

\section{II.B. $\quad \Delta V$ Requirements, Mission Phases, and Mission Timeline}

A mission architecture was created in such a manner as to minimize the overall weight required, which reduces the number of launches required for the mission, which in turn minimizes the overall cost of the mission. The chosen mission architecture requires several parts of the mission to be launched years before the astronauts launch, and then the astronauts will rendezvous with these supplies before heading to the target destination.

The Cryogenic Propulsion System (CPS), Space Exploration Vehicle (SEV), and Deep Space Habitat (DSH) will launch several years before the crew launches, and will utilize a spiral transfer orbit from low Earth orbit (LEO) to a highly elliptical orbit (HEO), using solar electric propulsion (SEP). This propulsion and mission architecture choice is justified in Section III. The crew will nominally launch from Earth August 11, 2025 using the Space Launch System (SLS) $100 \mathrm{mt}$ launch vehicle to get to LEO. After staying in a 400 $\mathrm{km}$ altitude LEO for a brief amount of time, they will then perform a burn to achieve $\Delta V=3.02 \mathrm{~km} / \mathrm{s}$, to rendezvous with the cargo and habitat in HEO. From HEO perigee, the cryogenic propulsion stage will provide $\Delta V=0.220 \mathrm{~km} / \mathrm{s}$ for a burn on August 23, 2025, which will put the spacecraft on a hyperbolic trajectory to arrive at $1999 \mathrm{AO} 10$ on December 31,2025 . Next, a burn using $\Delta V=2.194 \mathrm{~km} / \mathrm{s}$ will put the spacecraft on orbit with the asteroid for 14 days. On January 14, 2026, a burn of $\Delta V=1.450 \mathrm{~km} / \mathrm{s}$ will send the spacecraft on a return trajectory to Earths orbit, arriving March 3, 2026, to return to the Earth at a re-entry velocity of $11.25 \mathrm{~km} / \mathrm{s}$. A summary of the $\Delta V$ for the entire mission is shown in Figure 1, and an image of the completed trajectory is shown in Figure 2.

The total $\Delta V$ from HEO as a function of round-trip time and departure date is described in Figure 3(a), and illustrates launch window capability for the Vault-1 mission. Each $\Delta V$ is calculated with a given Earth departure date, round-trip duration and asteroid stay time. The asteroid arrival date is optimized to minimize the total $\Delta V$ from HEO. 


\begin{tabular}{|l|c|}
\hline \multicolumn{2}{|l|}{ First launch - DSH, CPS1, SEV } \\
\hline Mission phase & $\Delta \mathrm{V}[\mathrm{km} / \mathrm{s}]$ \\
\hline Launch $\rightarrow$ LEO & 9.80 \\
\hline LEO $\rightarrow$ HEO & 3.25 \\
\hline
\end{tabular}

\begin{tabular}{|l|c|}
\hline \multicolumn{2}{|c|}{ Second launch - MPCV, CPS2, crew } \\
\hline Mission phase & $\Delta \mathrm{V}[\mathrm{km} / \mathrm{s}]$ \\
\hline Launch $\rightarrow$ LEO & 9.80 \\
\hline LEO $\rightarrow$ HEO & 3.03 \\
\hline
\end{tabular}

\begin{tabular}{|l|c|}
\hline \multicolumn{2}{|l|}{ Docked components } \\
\hline Mission phase & $\Delta \mathrm{V}[\mathrm{km} / \mathrm{s}]$ \\
\hline HEO $\rightarrow$ escape trajectory & 0.22 \\
\hline Arrive at NEO & 2.19 \\
\hline Depart NEO & 1.45 \\
\hline
\end{tabular}

Figure 1. $\Delta V$ summary for the different aspects of the mission.

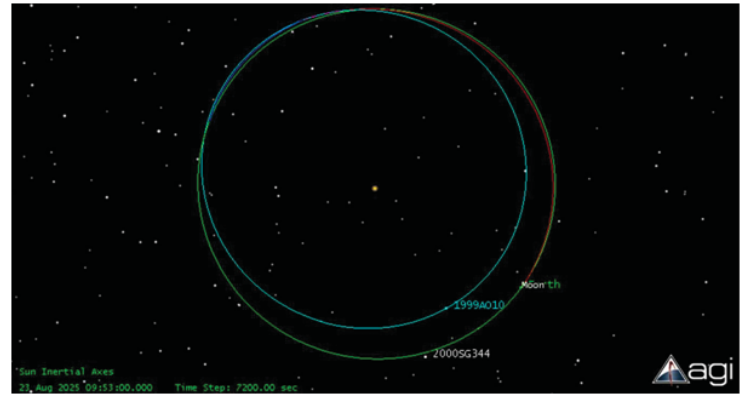

(a)

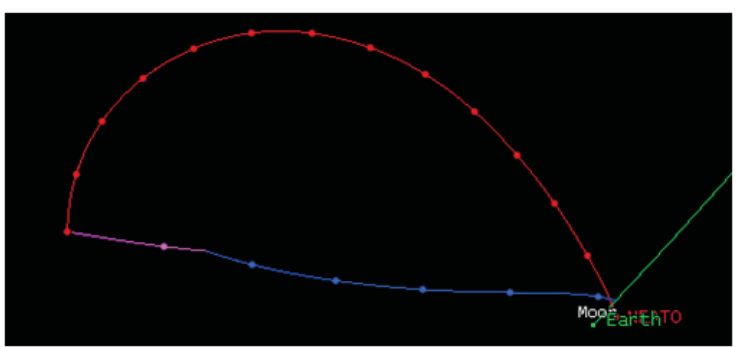

(b)

Figure 2. Trajectory from Earth to 1999AO10 and back (a); Earth centered view (b).

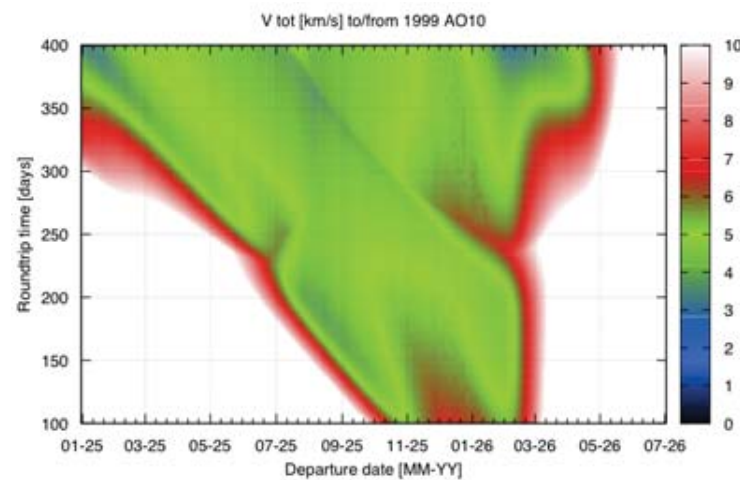

(a)

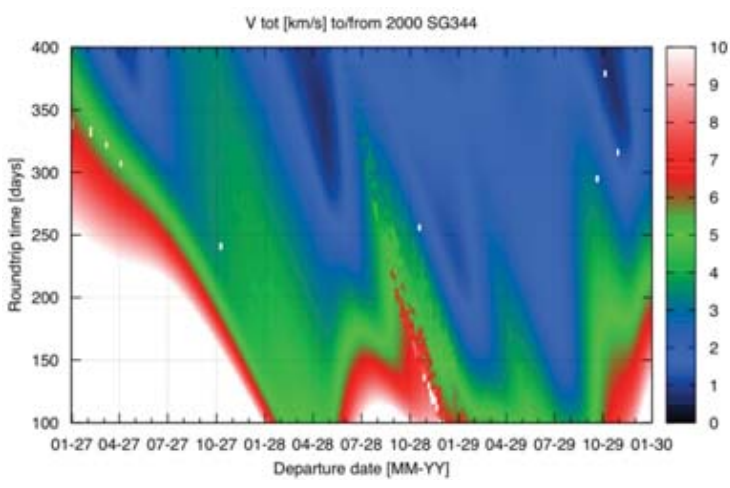

(b)

Figure 3. Total $\Delta V$ from HEO as a function of the Earth departure date and round-trip duration for (a) 1999 AO10 and (b) 2000SG344. 
The round-trip duration between the Earth departure and the target is required to be no greater than approximately 200 days. The round-trip duration is mainly limited due to human factors, which will be discussed in greater detail in Section V. The time spent at the asteroid is specified to be 14 days, to give the astronauts ample time to explore the asteroid and demonstrate the extra-vehicular activity (EVA) technology. The launch window will open in August 2025. A one week long launch window was desired at minimum, and the corresponding $\Delta V$ required from HEO to the target asteroid is $3.81 \mathrm{~km} / \mathrm{s}$, as shown in Figure $3(\mathrm{a})$. The launch window from HEO will be open between 17/8/2025 and 24/8/2025.

Asteroid 2000SG344 is the backup target for our mission. The total $\Delta V$ plot of 2000SG344 is described in Figure 3(b). The launch window will open in April 2028 and 2029, assuming that the round-trip duration is around 180 days.

In the event of a major accident, the abort capability of the mission was investigated for the baseline trajectory to 1999AO10, and this is shown in Figure 4. As expected, the $\Delta V$ requirement becomes larger if a quicker return to Earth is required and if the mission is aborted further from the launch date. The $\Delta V$ capability for Vault-1 on the outbound trajectory is $3.64 \mathrm{~km} / \mathrm{s}$ ( $\Delta V$ for the asteroid rendezvous and departure), which allows for a return to Earth at any time with a travel time under 40 days. If a faster return duration is required, the abort cut-off day of outbound trajectory is limited. For example, 10 days after Earth departure is the limit for a 5-day return duration. For all of the abort options considered, the Earth re-entry velocity is less than $12 \mathrm{~km} / \mathrm{s}$.

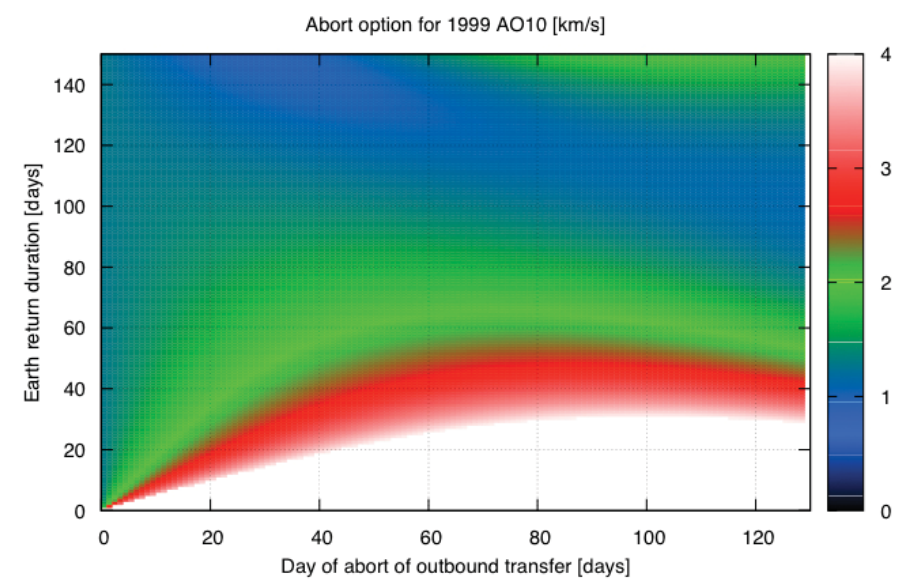

Figure 4. $\Delta V$ requirement for abort as a function of abort day from outbound transfer .

\section{Propulsion Systems}

Propulsion systems are crucial to the success of any space mission. Especially for a long-duration, manned mission, the propulsion system chosen needs to be extremely reliable and safe. Taking into account the strict mission constraints, detailed research on each of the components was performed and the best technology available was chosen. Different mission profiles were investigated, and this allowed the most efficient propulsion system for this mission to be chosen.

The driving factor for the propulsion system and mission profile selection was the choice of the target asteroid itself. This mission destination specified the required $\Delta V$, launch date, and the number of launch windows. This section assumes that there will be some reasonable technology advancement by the time that the mission will launch. In addition, the duration of the mission is constrained by human factors, crew safety, and the basic mission objectives, which had a large influence on the propulsion system chosen. The end design took advantage of an assumed international collaboration, and put an emphasis on developing and testing technologies that will be useful for future exploration of the Solar System.

\section{III.A. Propulsion Choice}

A modular approach was taken when trying to design this mission. The most advanced propulsion elements from around the world were incorporated into the design, and a strong emphasis was placed on interna- 
tional collaboration in order to make this mission feasible. Both cryogenic propulsion and the solar electric propulsion are considered for the two potential mission profiles.

The Cryogenic Propulsion Stage (CPS) was chosen to be a European Vinci Engine. Due to its high performance characteristics (vacuum thrust of $180 \mathrm{kN}$ and a specific impulse of $465 \mathrm{~s}$ ), this engine ensures that there will be sufficient thrust for both the crew and cargo modules. Since the propellant requirements for both the launches and the orbit insertion are different, two different scaled versions of the CPS have been used. With respect to the structure mass of the stage, a stage fraction of $15 \%$ has been assumed. Both of the CPS units contain active cryogenic fluid management and their own power management systems. In order to minimize propellant consumption, both CPS units take advantage of zero boil-off technology.

The Solar Electric Propulsion stage will use a Hall Thruster with Xenon gas as the propellant and a Stretched Lens Array Square-Rigger solar array (technology being developed currently), all of which are to be designed in the USA. This type of propulsion can be operated in different modes. In order to allow for a high payload insertion into HEO, a specific impulse of $5500 \mathrm{~s}$ for orbit insertion from LEO to HEO was selected. Despite the drawback of a high transfer time for the cargo from LEO to HEO (3.78 years), this solution enabled a large reduction in the amount of weight that needed to be launched from the Earth. This mass savings is mainly due to the low dry mass $(8.86 \mathrm{mt})$ for the propulsion system, and a wet mass of $19.92 \mathrm{mt}$, enabling a payload mass of $80.08 \mathrm{mt}$, for a $100 \mathrm{mt}$ Cargo Heavy Lift Launch Vehicle (HLLV) into HEO. The required power for this system is $200 \mathrm{~kW}$, which was chosen taking into account the relationship between the SEP specific impulse available, and the time required to travel from LEO to HEO, the effective payload, the propellant mass, and the inert mass. These comparisons are shown in Figure 5.

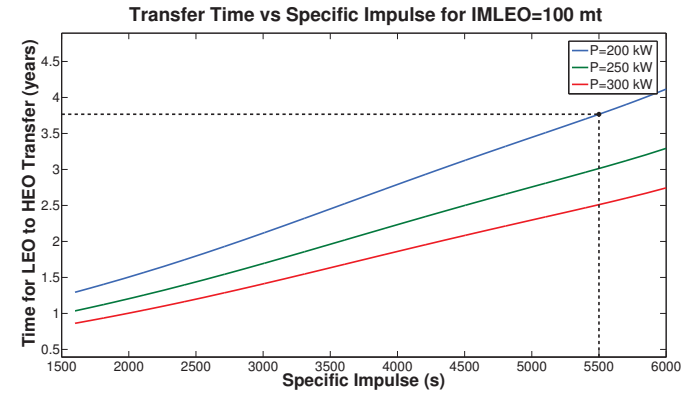

(a)

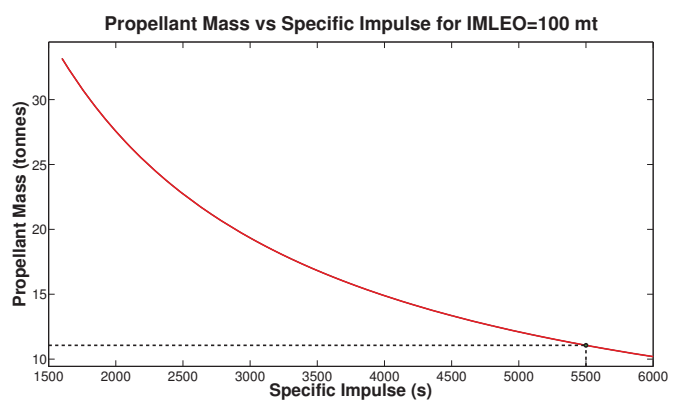

(c)

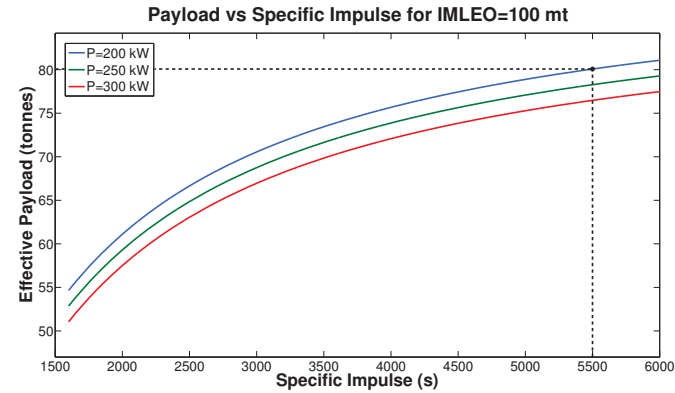

(b)

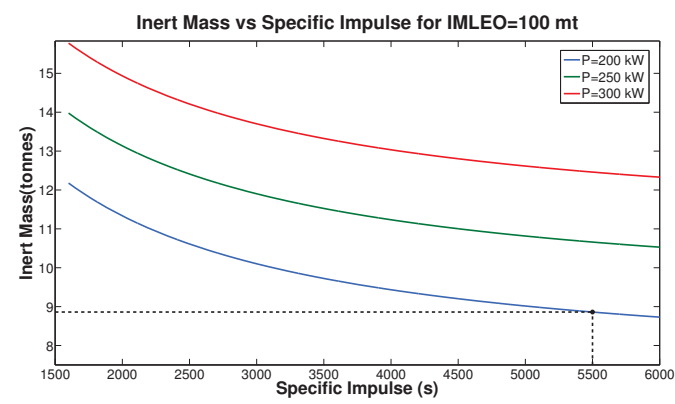

(d)

Figure 5. Power requirements: Time for LEO to HEO (a), Effective Payload (b), Propellant Mass (c), Inert Mass vs Specific Impulse (d), for $\mathrm{P}=200 \mathrm{~kW}$ (blue), $\mathrm{P}=250 \mathrm{~kW}$ (green), $\mathrm{P}=300 \mathrm{~kW}$ (red). The black dashed line shows the chosen operating conditions.

The USA Multi-Purpose Crew Vehicle (MPCV) designed for long duration deep space missions and a custom designed Deep Space Habitat (DSH) were chosen for the crew to live in. As well as the CPS, this element includes connection adapters necessary to dock with SEV, CPS and MPCV. Another important component, the SEV, was chosen to be designed in Japan and is designed to land on the NEO's surface carrying two astronauts and scientific equipment. Lastly, the European Kick Stage is used to deliver an additional velocity increment required to put the spacecraft on the required trajectory. 


\section{III.B. Mission Profiles}

There are two feasible mission profiles that were considered when determining the optimal propulsion system. The first option uses strictly chemical propulsion, whereas the second option uses a combination of chemical and solar electric propulsion. Both of these mission profiles are described below.

\section{III.B.1. Mission Profile 1: Chemical Scenario}

In this scenario, two launches are required to reach LEO (Figure 6). The first launch carries a payload that includes the CPS-1, DSH, and SEV. The second launch puts the MPCV and CPS-2 into LEO at a $407 \mathrm{~km} \mathrm{x}$ $407 \mathrm{~km}$ orbit. Both cargo and crew then reach a $407 \mathrm{~km}$ x 233,826 km highly elliptical orbit (HEO) with a 5 day period using chemical propulsion. The second launch uses a kick stage, which is jettisoned after HEO orbit insertion. Once arrived at HEO, all of the elements are docked and the stack becomes a Deep Space Vehicle (DSV). The transfer to NEO is fueled by the CPS-2, that is later jettisoned in space before reaching the asteroid. The final approach is completed using the CPS-1.

At the target location, the DSV is inserted in an orbit around the target asteroid, and scientific activities are undertaken for 14 days. On the return journey to earth, propulsion is provided by the CPS-1, and the crew capsule returns to the Earth. As soon as the capsule is in Earth's vicinity, the DSH is released in space.

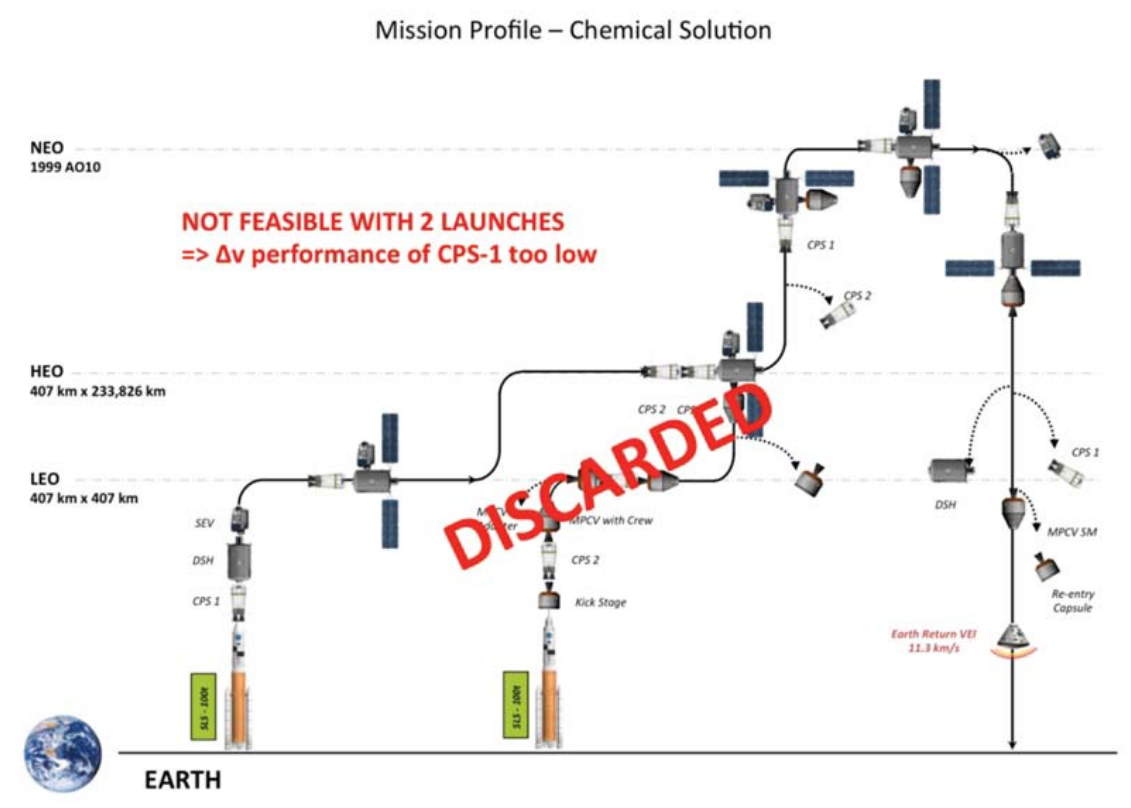

Figure 6. Chemical mission profile.

\section{III.B.2. Mission Profile 2: Hybrid Scenario}

In this scenario, a combination of chemical and solar electric propulsion is proposed (Figure 7). The same orbits used in the previously described mission profile are used again. Two launch vehicles are used to bring the necessary modules to LEO. The first launch contains the CPS-1, the SEP stage, the DSH, and the SEV. The second launch, which is at a much later date, contains a kick stage, the CPS-2 and the MPCV, along with the crew. Due to its low thrust, the use of the SEP stage to reach HEO requires an earlier departure of the cargo components. It is estimated that the cargo will take 3.78 years to reach HEO from LEO, using a spiral trajectory and electric propulsion. After the spiraling phase has been completed, the SEP stage is released into space.

\section{III.C. Final Propulsion Selection}

Although both of the mission profiles theoretically comply with the mission requirements, the final choice of the propulsion system was highly driven by cost considerations. 


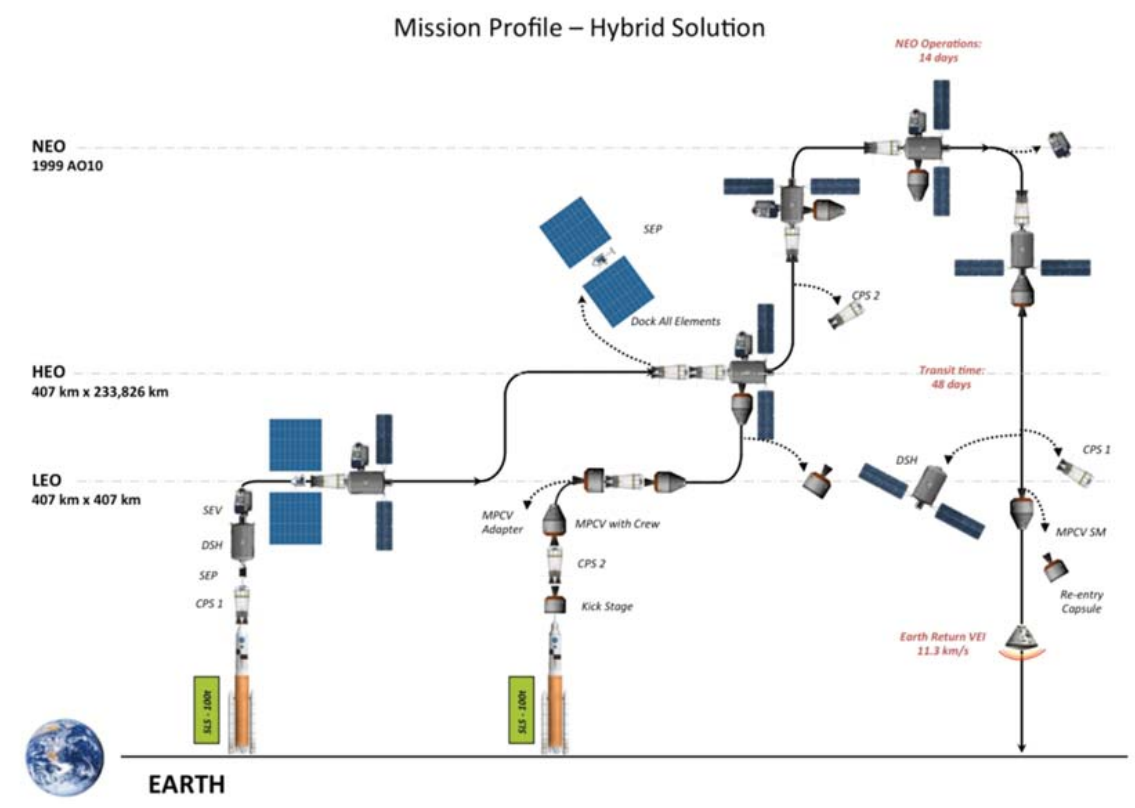

Figure 7. Hybrid mission profile.

Comparing both cases, it can be concluded that using chemical propulsion substantially increases the cost of the mission due to the need of larger, or even multiple launch vehicles. After comparing the previously discussed mission profiles, the results clearly show that it is not possible to complete this mission with only two launches, if only chemical propulsion is used. The mass savings due to the exploitation of SEP have been proven during many earlier missions. In the Dawn mission, the mission cost was reduced by $50 \%{ }^{3}$ Additionally, using solar electric propulsion allows the mission to be much more flexible.

On the other hand, the chemical scenario has the advantage of following a much simpler trajectory, as electric propulsion requires the use of a more complicated trajectory, and the time of flight also increases significantly. This results in a longer time spent in the Van Allen radiation belt for any module that is launched with the SEP stage. If humans were launched with this stage, it would require much additional protective shielding, and this radiation can also easily damage any equipment that is sensitive to radiation. Therefore, using SEP in a human mission to an asteroid is economical and feasible as long as it is only used only for cargo transfer from LEO to higher orbits.

In conclusion, the hybrid mission profile assures maximal savings by reducing the number of launches, which accounts for a major part of the mission cost. Moreover, this scenario fulfills the need for developing SEP technology, which would be useful for future deep space mission as well. Therefore, the mission profile chosen for the Near-Earth asteroid mission is the hybrid scenario, that uses chemical propulsion for transporting crew and solar electric propulsion for cargo to reach HEO.

\section{Science Experiments}

Having examined the trajectory and propulsion system needed to reach the asteroid, the various science payloads that will be carried on-board in order to meet the science objectives of the mission are described in this section. The various sensors and their functions are described in Table 2. 
Table 2. List of all the science instruments and measurements.

\begin{tabular}{|c|c|c|}
\hline Instrument & Experiment & Description \\
\hline Optical Camera & Imaging & $\begin{array}{l}\text { The camera will take high resolution images of the as- } \\
\text { teroid that will also help determine its spin state. }\end{array}$ \\
\hline NIR Spectrometer & Mineralogy & $\begin{array}{l}\text { The near-IR spectrometer will give information about } \\
\text { the composition of the asteroid. }\end{array}$ \\
\hline TIR Spectrometer & Thermal Mapping & $\begin{array}{l}\text { The thermal infrared spectrometer will allow us to gen- } \\
\text { erate a thermal map of the asteroid surface, in addition } \\
\text { to giving information about the composition of the as- } \\
\text { teroid. }\end{array}$ \\
\hline $\begin{array}{l}\text { Vis-IR camera, } \\
\text { Thermal Conductivity } \\
\text { Probe }\end{array}$ & Internal Structure & $\begin{array}{l}\text { The astronauts will dig holes into the surface of the } \\
\text { asteroid to insert these probes. These measurements } \\
\text { will give us information about the thermal properties } \\
\text { of the asteroids interior. }\end{array}$ \\
\hline Laser Altimeter & Topography & The altimeter will generate a 3D map of the asteroid. \\
\hline $\begin{array}{l}\text { Ground Penetrating } \\
\text { Radar }\end{array}$ & Subsurface Imaging & $\begin{array}{l}\text { This microwave radar will give information about the } \\
\text { internal structure of the asteroid. }\end{array}$ \\
\hline Dust Detector & Dust Environment & $\begin{array}{l}\text { The impinging solar wind can differentially charge the } \\
\text { surface, causing dust particles on the surface to levitate. } \\
\text { Their population will be studied by this instrument. }\end{array}$ \\
\hline Magnetometer & Magnetic Field & $\begin{array}{l}\text { While asteroids are expected to be unmagnetized bod- } \\
\text { ies without an atmosphere, the interaction with the so- } \\
\text { lar wind can cause wake effects and other interesting } \\
\text { perturbations in the background Interplanetary Mag- } \\
\text { netic Field (IMF) which will be studied with SQUID } \\
\text { (Superconducting Quantum Interference Device) mag- } \\
\text { netometers. }\end{array}$ \\
\hline $\begin{array}{l}\text { Radiation Assessment } \\
\text { Detector }\end{array}$ & $\begin{array}{l}\text { Radiation } \\
\text { Environment }\end{array}$ & $\begin{array}{l}\text { This sensor will be active during the onward and return } \\
\text { journeys and will be used to assess the radiation levels } \\
\text { in the interplanetary medium. }\end{array}$ \\
\hline Geophones & Seismology & $\begin{array}{l}\text { Geophones are seismic sensors that will be used for } \\
\text { studying the internal structure of the asteroid. The } \\
\text { astronauts will plant explosives on the asteroid and det- } \\
\text { onate them on their way out to study the resulting seis- } \\
\text { mic waves. }\end{array}$ \\
\hline $\begin{array}{l}\text { X-Ray Fluorescence } \\
\text { Spectrometer }\end{array}$ & $\begin{array}{l}\text { Elemental } \\
\text { Composition }\end{array}$ & $\begin{array}{l}\text { The X-rays from sun can cause the asteroid surface to } \\
\text { fluoresce, the spectrum of which contains valuable in- } \\
\text { formation about asteroid composition. }\end{array}$ \\
\hline
\end{tabular}


In addition to the experiments listed in Table 2, a gravitational map of the asteroid will be generated using the Delta Differential One-Way Ranging (DOR) technique. With this technique, the ranging will be carried out using two radars from two different locations on earth and precise orbit of the spacecraft can be obtained by comparing their measurements. However, in order for this to succeed, we need to eliminate the effects of the ionosphere, which can be done by calibrating against a standard source like a quasar (Figure 8). This experiment can be carried out using the communication antennas on the spacecraft and hence no additional payload is required for this measurement. ${ }^{4}$

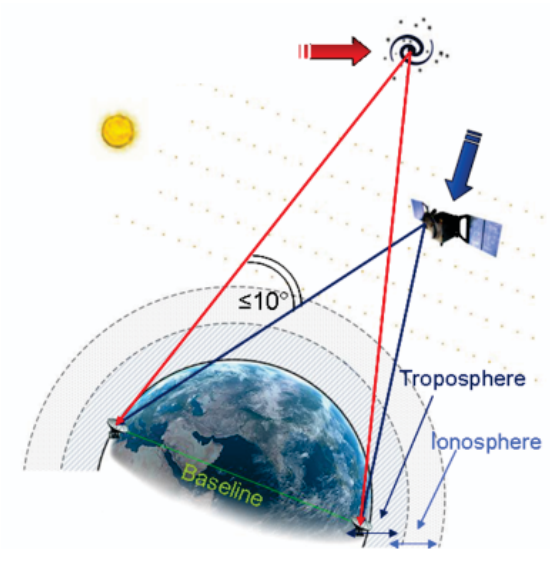

Figure 8. An illustration of Delta DOR.

The measurements being carried out by the various sensors listed previously broadly fall into the following four categories:

1. Physical Properties: Optical Camera, Laser Altimeter, Thermal Infrared (TIR) Spectrometer, Delta DOR

2. Composition: Near Infrared (NIR) Spectrometer, TIR Spectrometer, X-ray Fluorescence Spectrometer

3. Internal Structure: Geophones, Thermal Conductivity Probe, Ground Penetrating Radar

4. Environment: Radiation Assessment Detector, Dust Detector, Magnetometer

These experiments, in combination with samples returned by the astronauts, will lead to the complete characterization of the asteroid, generating a wealth of information for scientific investigation.

\section{Human Factors}

As this mission would be the first deep-space human mission, there are many unknowns that add much complexity to the mission design. It is essential to consider the physical and psychological well-being of the crew before making design decisions. A manned mission is not without risk, but it is critical to minimize the danger in all elements. The crew habitat must be designed in such a way to keep the astronauts healthy and happy, while still maintaining a cost and complexity level such that the mission is feasible.

A variety of physical and psychological factors were considered in the design of the deep space habitat, with pre-existing NASA requirements addressed. Driving factors include: crew size; mission duration; life support; radiation effects; micro-gravity effects; and psychological effects.

\section{V.A. Crew Size and Mission Duration}

In order to begin to design the habitable areas for the crew, it is necessary to determine crew size and mission duration. Based on these factors, habitat size, weight, critical systems, etc. can be designed to support the crew.

Current NASA specifications state that an acceptable risk of radiation exposure on any mission is such that there is a "limit of $3 \%$ fatal cancer risk at a $95 \%$ confidence level." The interaction between humans and 
Galactic Cosmic Rays (GCRs) is not well understood, and therefore acceptable radiation exposure levels are an educated guess, at best. Because of this, a significant safety factor must be included to help mitigate the risks to the astronauts for radiation exposure. This was one of the main drivers that limited the allowable mission duration to approximately 200 days. After this was determined, it was decided that a crew selection of 3 people would be acceptable for the mission. 3 crew members allows for some human redundancy to exist, while also keeping the overall mission weight to a minimum. Through careful crew selection, we believe that an all-male crew of target age 50 is most appropriate for this mission to mitigate radiation effects. ${ }^{5}$ Successful applicants for this mission will be required to have a wide variety of skills, placing a high emphasis on medical training.

\section{V.B. Deep Space Habitat Design}

The crew habitat for this mission is based on the NASA Deep Space Habitat (DSH) module. ${ }^{6}$ In addition to the DSH, there will also be a MPCV that is connected to the DSH. The total internal volume of these two spaces accounts for the total livable space for the astronauts. All numbers were linearly scaled from the NASA DSH module to try to accurately estimate weight, power costs, total size etc., for the habitat. Several studies have been performed trying to determine how much space is needed per person on a mission of this type to allow the astronauts to continue to function "optimally." ${ }^{2}$ For this mission, $18.0 \mathrm{~m}^{3}$ of habitable volume per person was determined to be a fair balance between human performance and weight/cost constraints.

A mission length of 200 days falls on the cusp where an open-loop life support system and a closed-loop life support are almost equally efficient. ${ }^{7}$ For this mission, a partially closed life support system is proposed. Waste water recycling will be used as this technology is more mature and has been used previously on the ISS, ${ }^{8}$ and the waste water storage containers can be strategically placed in the habitat to offer additional radiation shielding for the astronauts. The thermal and power systems used are fairly standard in nature, and should be easily powered by a solar array. Power consumption estimates and thermal control systems are very similar for both the DSH and the MPCV. Redundant subsystems, contingency plans, and abort scenarios have also been considered throughout the design process.

With the basic architecture now defined, many other factors were identified and addressed in the design. Primarily, these include radiation effects, micro-gravity effects, and psychological effects.

\section{V.C. Radiation}

In order to best regulate the level of radiation that the crew will be exposed to on a mission such as this one, a three level plan was developed. This includes strict monitoring of the astronauts, radiation shielding for the habitat, and a warning system for Solar Particle Events (SPEs).

Monitoring involves the astronauts being required to constantly wear dosimeters, as well as being required to report radiation exposure levels regularly to mission control.

The radiation shielding involves some standard aluminum shielding in the outer habitat wall $\left(10 \mathrm{~g} / \mathrm{cm}^{2}\right)$, as well as efficient packing of supplies. It has been shown that creative packing of supplies near the external walls of the habitat ${ }^{2}$ (consumables, cargo, etc) can significantly reduce radiation dosage received by the crew. In addition, the crew quarters will have water shielding and this area will act as a shelter should an SPE occur during the mission.

Finally, the warning system involves either advance notice from NASA, or data from satellites, to warn the astronauts of an impending SPE and allow them to get to their quarters in a timely manner. Preliminary calculations were performed assuming one SPE (strength based on the August 1972 event) during the mission, and, not taking into account the added benefit of efficient packing, suggest that the astronauts will be well within the acceptable level of radiation dosage prescribed by NASA.

\section{V.D. Microgravity}

It is known that prolonged exposure to a micro-gravity environment has negative effects on the cardiovascular system, muscular physiology, bone physiology, and vestibular physiology. Some of the negative effects have been studied based on previous missions, as well as through extended stays on the ISS. These negative effects will be countered through extensive exercise routines, such as those currently employed on the ISS. For a mission of 200 days, a method to create artificial gravity has been deemed not critical. However, the 
combined effects of radiation exposure and microgravity have never been studied before, and this will have to be monitored throughout this mission.

\section{V.E. Psychology}

In addition to these physical factors, there are many psychological factors that must be taken into account. For example, separation from family for a long duration, absence of privacy, and isolation and confinement are just a few of the hardships that the astronauts will have to undergo throughout this mission. If not accounted for, these factors can seriously decrease a person's ability to function efficiently and could put the mission in jeopardy. In order to keep the crew operating at peak performance, many precautions will be taken. Some of these include: crew selection, pre-launch training (stress coping, effective communication, team building), mental status monitored by mission control (during mission), exercise and work routines, and communication with family members. Through careful planning and monitoring, it will be possible to keep the astronauts efficient and capable throughout the entire mission.

\section{Conclusion}

The Vault-1 Mission shows that it would be feasible to successfully complete a manned mission to a Near Earth Object by the year 2025. The above mission proposal makes conservative estimates on technologies to be developed, and assumes a large amount of international collaboration. A balance was achieved between using pre-existing, reliable technologies and incorporating new technologies that would be needed for longerterm deep space human missions. Vault-1 would serve as a stepping stone in the path of human deep space exploration, and would be a great accomplishment on the path towards a human mission to Mars. Finally, this mission proposal demonstrates the importance of short, high-intensity student competitions. After 5 days of work, both teams participating in the Caltech Space Challenge produced detailed proposals outlining the feasibility of a manned asteroid mission, while also providing in-depth technical detail regarding its implementation. A successful international human mission to a Near Earth Object would greatly enhance technical and scientific knowledge, while helping improve international relations and inspiring the whole of humanity.

\section{Acknowledgments}

This study was conducted as a part of the 2011 Caltech Space Challenge, a week long workshop/competition directed towards the U.S.A Presidents initiative of sending astronauts to an asteroid by 2025. This challenge worked to address the 2010 United States National Space Vision, as well as satisfying growing international interest in the exploration of Near Earth Objects. The challenge was hosted by the Keck Institute for Space Studies (KISS), and held at the California Institute of Technology from Sept 12-16, 2011. The event was organized by two graduate students studying at the California Institute of Technology, Prakhar Mehrortra and Jonathan Mihaly. The event was managed by Michele Judd, the Managing Director for the Keck Institute for Space Studies, and the student organizers were mentored by Dr. Donald Yeomans, John D. Baker, and Professor Paul E. Dimotakis. 32 international participants representing 12 nations were invited to participate in the challenge after two rounds of selection process. The participants were divided into two teams - Team Voyager and Team Explorer, and were challenged to prepare competing proposals for the first manned mission to a Near Earth Object.

We would like to acknowledge the contributions of our team members Emmet M. Cleary, Aditi J. Dighe, Ken P.L. Ho, Arlen Kam, Paola Libraro, Daniel Y. Lo, Pascal Renten, Apoorv Sharma, Michael A. Skeen, Tomohiro Yamaguchi and Samuel R. Zarovy, and our project mentors Dr. Paul E. Dimotakis, Dr. Donald K. Yeomans, Nigel Angold, Nathan Strange and Aline Zimmer. Special thanks also go to Michele Judd, for making this event possible. In addition we would like to thank the sponsors of this event, Lockheed Martin, SpaceX, Orbital Sciences Corporation, Analytical Graphics Inc., Mrs. Helen Keeley, Dr. Louis J. Alpinieri, and Dr. Fernando Fernandez. 


\section{References}

${ }^{1}$ Abell, P., "Near-Earth Objects: Targets for Future Human Exploration, Solar System Science, and Planetary Defense," Public Lecture - Caltech Space Challenge 2011 http://kiss.caltech.edu/workshops/space-challenge2011/media/abell/1_ abell.html, 2011.

${ }^{2}$ Hopkins, J., Dissel, A., Jones, M., Russel, J., and Gaza, R., "Plymouth Rock: An Early Human Mission to Near Earth Asteroids Using Orion Spacecraft," Lockheed Martin Corporation, 2011.

${ }^{3}$ Brophy, J. R., Gershman, R., Strange, N., Landau, D., Merrill, R. G., and Keslake, T., "300-kW Solar Electric Propulsion System Configuration for Human Exploration of Near-Earth Asteroids," 47th AIAA/ASME/SAE/ASEE Joint Propulsion Conference and Exhibit, AIAA, 2011.

${ }^{4}$ Maddè, R., Morley, T., Abelló, R., Lanucara, M., Mercolino, M., Sessler, G., and de Vicente, J., "Delta-DOR: A New Technique for ESAs Deep SpaceNavigation," ESA Bulletin (ISSN 0376-4265), 2006.

${ }^{5}$ Cucinotta, F. A., Hu, S., Schwadron, N. A., Kozarev, K., Townsend, L. W., and Kim, M.-H. Y., "Space radiation risk limits and Earth-Moon-Mars environmental models," Space Weather, Vol. 8, 2010.

${ }^{6}$ Toups, L., Smitherman, D., Shyface, H., Simon, M., Bobskill, M., Komar, D. R., Guirgis, P., Bagdigian, B., and Spexarth, G., "Deep Space Habitat Team: HEFT Phase 2 Efforts," NASA Internal Report, 2010

${ }^{7}$ Hurlbert, K., Bagdigian, B., Carroll, C., Jevarajan, A., Kliss, M., and Singh, B., "DRAFT Human Health, Life Support and Habitation Systems," NASA Internal Report, 2010.

${ }^{8}$ Levine, H. G. and Roberts, M. S., "Forward Osmosis Bag (FOB)," NASA - http://www.nasa.gov/mission_pages/ station/research/experiments/FOB.html, 2012. 